\title{
Evaluación de la matriz de IPEBA para la acreditación de las instituciones de Educación Básica Regular*
}

\section{Evaluation of IPEBA's matrix for the accreditation of the basic education institutions}

Recibido: 16-06-2015 Aceptado: 08-07-2015

Palabras clave: calidad, acreditación, estándares, autoevaluación, IPEBA.

Keywords: quality, certification, standards, selfassessment, IPEBA.

Maeve Rosana Marroquin Gomez

Colegio FAP José Quiñonez maeverosana@yahoo.com Mónica Lévano Ochoa Telesup monicalevanoochoa@gmail.com

\section{Maeve Rosana Marroquin Gomez Mónica Lévano Ochoa}

RESUMEN. Se realizó un estudio con el fin de evaluar la matriz de IPEBA para la acteditación a la calidad educativa de las instituciones de Educación Básica Regulat. Ed objetivo fue conocer en qué medida los estándares de IPEBA cumplen con los requerimientos de calidad, sus características y su adecuación pata orientar y evaluar el proceso de enseñanza aprendizaje.

Participaron 15 expertos en educación segmentándose en tres grupos (líderes, académicos y directores), a quienes se les aplicó un cuestionario. El trabajo permitió responder a las preguntas de la investigación. Los resultados conllevan* a concluir que la propuesta de IPEBA no alcanzó el nivel de concordancia establecido como aceptable (60\%), aunque está muy cerca $(57.3 \%)$.

ABSTRACT. A study has been conducted to evaluate IPEBA's matrix for the educational quality certification for Regular Basic Education institations. It was intended to determine to what extent IPEBA's standards met quality requirements, its characteristics, and adaptation to guide and evaluate the teaching/learning process. A survey was applied to fifteen educational expetts that were parted into three groups (leaders, academics, and headmasters). This allowed investigation inquiries to be answered. Results concluded that IPEBA's proposal was unable to reach the concordance level established as acceptable $(60 \%)$, although it stands very close $(57.3 \%)$.

* Tesis para optar el Grado de Magister en Educación, UNIFE, 2015 


\section{Introducción}

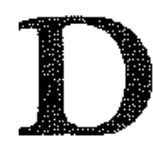

urante los últimos años existe una preocupación permanente por la educación de todos los actores que intervienen y se enfrentan a los resultados del proceso educativo. Cuando se va avanzando en ella, aparecen nuevas expectativas más dificiles de alcanzar convirtiéndose la calidad en una carrera continua de mejota constante de evaluación, actualización e innovación. La calidad es un factor estratégico, clave del que dependen la mayor parte de las organizaciones no sólo para mantener su posición en el metcado, sino incluso para asegurar su supervivencia.

Álvarez (2006) sostiene que "calidad presenta un proceso de mejota continua, en la cual todas las áreas de la empresa buscan satisfacer las necesidades del cliente o anticiparse a ellas, participando activamente en el desarrollo de productos o en la prestación de servicios".

La educación se ha convertido en un instrumento potencial de progreso económico y social, que busca la forma de mejorar la calidad, brinda alcances y utiliza estrategias e instrumentos para que el producto no solo sea competitivo intelectualmente, sino que sea crítico, inclusivo, participativo e integral.

Hay una corriente internacional importante hacia la acreditación a la calidad educativa. Bajo esta propuesta, el gobierno peruano creó el Instituto Petuano de Evaluación, Acreditación y Certificación de la Calidad de la Educación Básica Regular (IPEBA) cuyo propósito es articular esfuerzos hacia el logro de aprendizajes y la formación integral de los estudiantes. La acreditación ayuda a certificar el nivel de educación que se está impartiendo en las instituciones educativas lo cual es beneficioso para los estudiantes, los padres de familia y la sociedad ya que así se sabe que podemos esperar de la educación que se está impartiendo. Por ello, es útil que las instituciones educativas se acrediten. Sin embargo, no hay que perder de vista el objetivo que consiste en dar una mejor calidad de educación, que sea más útil a los estudiantes y a la sociedad, y ello se consigue a través de la mejota de los procesos educativos tanto
Alétheia 2015,3(1) 73

de enseñanza aprendizaje como administrativos para lo cual se debe tener presente que estamos en un mundo pluricultural y globalizado.

El trabajo del IPEBA se orienta a promover una amplia participación y debate, que involucte a todos los actores para que tengan la oportunidad de exponer sus puntos de vista y llegar a acuerdos aceptados por la sociedad.

Sin embargo, la acreditación debe entenderse como un proceso de mejora continua que comprende tres etapas:

La primera consiste en la autoevaluación que realiza la propia IE, con la finalidad de reflexionar sobre los aspectos que facilitan y dificultan sostener la mejora continua del proceso de enseñanzaaprendizaje. La autoevaluación no es autorreferencial. Se orienta por la matriz de evaluación propuesta que facilita la identificación de fortalezas y debilidades en la gestión de la IE para tomar decisiones de mejora. Esta etapa culnina en el plan de mejota que consiste en la planificación de acciones que surjan como propuestas, a partir del análisis de las causas de las principales situaciones encontradas. El proceso de autoevaluación realizado, los resultados, el plan de mejora elaborado y las lecciones aprendidas sobre el proceso se consolidan en un informe de autoevaluación que servitá de insumo para el proceso de evaluación externa. La matriz de evaluación está estructurada en factores, estándares e indicadores. Se entiende por factor a la variable o grupo de variables relacionadas a la gestión educativa de la institución, que incide en la mejora del proceso de enseñanzaaprendizaje. Estos factores han sido identificados a partir del recojo de percepciones sobre calidad educativa, realizado con distintos actores del pás (estudiantes, docentes, directores $y$ familias de instituciones educativas públicas y privadas) y la revisión de estudios de factores asociados a logros de aptendizaje, investigación sobre efectividad escolar, modelos de evaluación de educación básica y experiencias exitosas de mejora de la calidad educativa en el pais. El estándar precisa las expectativas respecto a la gestión educativa esperada y los indicadores son acciones observables y susceptibles de ser medidas 


\section{UNIFÉ - EPG}

que permiten determinar el nivel de cumplimiento de los estándares establecidos. Estos tres componentes se encuentran relacionados entre sí: los estándares se elaboran en función de los factores y los indicadores se elaboran en función de los estándares

La segunda etapa es la evaluación externa. La realiza una entidad evaluadora que cuenta con autorización del IPEBA, por lo que figura en el Registro de entidades evaluadoras con fines de acreditación. Se ejecuta en base a la matriz para la evaluación de la calidad de la gestión educativa, el informe de autoevaluación de la institución educativa y la información recogida durante el proceso de evaluación. La evaluación externa es solicitada por la institución educativa, aporta una mirada objetiva y ofrece recomendaciones al proceso realizado, brinda retroinformación sobre fortalezas, buenas prácticas y retos que deberá superar para fortalecer su capacidad de mejora continua del proceso de enseñanzaaprendizaje. Además, identifica las necesidades que tiene la institución educativa, señala el nivel de progreso alcanzado respecto a los estándares establecidos y presenta recomendaciones al IPEBA.

La tercera etapa es la acreditación propiamente dicha. Se otorga a partir del informe de la entidad evaluadora. La vigencia de la acreditación es temporal. Variará en función de cuan cerca se encuentra la institución educativa de contar con una gestión que permita la mejora continua del proceso de enseñanzaaprendizaje. El tiempo máximo de acreditación es de cinco años. Luego de este periodo, para renovarla, la institución educativa deberá implementar un nuevo proceso de autoevaluación y evaluación externa en la perspectiva de mejora continua como proceso dinámico (Morgan, 2011)

La propuesta de acreditación evalúa la gestión educativa para comprender qué procesos y estrategias internas ponen en práctica las instituciones educativas públicas y privadas cuando se enfrentan a la tarea de mejorar el proceso de enseñanza aprendizaje. Como toda evaluación, se limita a observar algunos aspectos, y se han seleccionado aquellos relacionados con la capacidad de gestión para mejorar los resultados educativos progresivamente con el fin de comprender la relación entre las acciones que realizan las instituciones educativas y los resultados que van obteniendo (Morgan, 2011).

\section{Método}

\section{Nivel y diseño de investigación}

El nivel de investigación es descriptivo y de tipo sustantivo y pretende describir las reacciones de sujetos relacionados a la educación desde diferentes responsabilidades y explicar dichos resultados. Por otro lado, el diseño es descriptivo simple. Es una investigación de naturaleza cualitativa.

\section{Participantes}

Se consideró 15 representantes de instituciones gubernamentales, universidades, instituciones educativas y miembros de organismos de cooperación; los mismos que se consideran muy importantes debido a la experiencia, estudios, reportes de trabajos relacionados y práctica en la actividad educativa.

\section{Instrumentos}

Para la recolección de las opiniones acerca de la matriz de IPEBA se empleó una guía con preguntas estructuradas abiertas centradas en el tema de investigación con un vocabulario acorde al nivel de los entrevistados. A través de juicio de expertos, líderes de opinión seleccionados, se evaluaron los cinco factores, doce estándares y 43 indicadores. Se relacionaton y se encuentraron los puntos de coincidencia y discrepancia por categoría de líderes de opinión. De esta contrastación, se seleccionó aquellas ocurrencias que merecían una mejor o mayor explicación.

\section{Técnicas de procesamiento y análisis de datos}

Para el análisis de los datos, se hizo uso de la estadística descriptiva y del análisis de contenido y valoración de los líderes por factor de tal manera que cada líder evaluó solo un factor y sus respectivos estándares e indicadores. Se ha estableció un nivel de concordancia aceptable del 60\% entre la propuesta de IPEBA y la posición de los expertos. 


\section{Resultados}

A continuación, se presentan los resultados obtenidos por cada factor, incluyendo los estándares que los constituyen. Así mismo, el resultado total (de toda la matriz).

Factor 1: Dirección institucional, está compuesto por tres estándares.

Tabla 1

Resumen de nivel de concordancia con el factor 1 de la propuesta de IPEBA

\begin{tabular}{lc}
\hline Estandar & \% de concordancia \\
\hline Total estándar 1 & 44.41 \\
Total estándar 2 & 51.10 \\
Total estándar 3 & 65.91 \\
\hline Total Factor & 53.08 \\
\hline
\end{tabular}

De los tres estándares, solo uno supera el nivel de concordancia aceptable (estándar 3); los otros dos están lejos de este criterio. Por ello, el factor 1 no alcanza el nivel de concordancia aceptable.

Factor 2: Soporte al desempeño docente, compuesto por tres estándares.

Tabla 2

Resumen del nivel de concondancia con el Factor 2 de la propuesta de IPEBA

\begin{tabular}{lc}
\hline Estandár & \% de concordancia \\
\hline Total estándar 4 & 66.82 \\
Total estándar 5 & 96.66 \\
Total estándar 6 & 62.21 \\
\hline Total Factor & 75.23 \\
\hline
\end{tabular}

De los tres estándares, uno de ellos supera, ampliamente, el nivel de concotdancia aceptable (estándar 5). Los ottos dos están sobre este criterio. Por ello el factor 2 alcanza el nivel de concordancia aceptable.

Factor 3: Trabajo conjunto con las familias y comunidad, compuesto por dos estándares.
Tabla 3

Alétheia 2015, 3 (1)

75

Resumen del nivel de concordancia con el Factor 3 de la propuesta de IPEBA

\begin{tabular}{lc}
\hline Estándar & $\%$ de concordancia \\
\hline Total estándar 7 & 49.99 \\
Total estándas 8 & 63.33 \\
\hline Total Factor & 56.66 \\
\hline
\end{tabular}

De los dos estándates, solo uno supera el nivel de concordancia aceptable (estándar 8). El otro está lejos de este criterio. Por ello, el factor 3 no alcanza el nivel de concordancia aceptado.

Factor 4: Uso de la información, compuesto por dos estándares.

Tabla 4

Resumen del nivel de concondancia con el factor 4 de la propuesta de IPEBA

\begin{tabular}{cc}
\hline Estándar & \% de concotdancia \\
\hline Total estándar 9 & 17.77 \\
Total estándar 10 & 31.11 \\
\hline Total Factor & 24.44 \\
\hline
\end{tabular}

Ninguno de los dos estándates supetà el nivel de concordancia aceptable. Por ello, el factor no alcanza el nivel de concordancia aceptablè.

Factor 5: Infraestructuta y tecutsos para el aprendizaje, compuesto por dos estándares.

Tabla 5

Resumen del nivel de concordancia con el factor 5 de la propuesta de IPEBA.

\begin{tabular}{lc}
\hline Estándar & \% de concordancia \\
\hline Total estándar 11 & 47.33 \\
Total estándar 12 & 90.00 \\
\hline Total Factor & 68.66 \\
\hline
\end{tabular}

De los dos estándares, uno está por debajo del nivel de concordancia (estándar 11) y el otro lo supera ampliamente (estándar 12). Por ello, el factor 5 alcanza el nivel de concordancia aceptable. 
76 UNTEÉ - EPG

Total: Toda la matriy

La tabla 6 presenta el resumen de los resultados totales de los 5 factores antes presentados.

Tabla 6

Puntaje de concordancia por factor

\begin{tabular}{lc}
\hline Estandar & \% de concordancia \\
\hline Total estándar 1 & 53.80 \\
Total estándar 2 & 75.23 \\
Total estándar 3 & 56.66 \\
Total estándar 2 & 24.44 \\
Total estándar 3 & 75.16 \\
\hline Total Factor & 57.05 \\
\hline
\end{tabular}

Puede observarse que el factor 4 está muy por debajo del nivel de concordancia y 2 de ellos 1 y 3 son cercanos, los factores 2 y 5 están por encima de éste $(60 \%)$.

\section{Discusión}

Los resultados indicaron que en general la propuesta de IPEBA tiene un nivel de concordancia de $57 \%$, muy cerca del nivel establecido. Esto está influenciado por los dos factores que lo elevan, 2 y 5 , que, en general, muestran la posibilidad de aplicación. No obstante, es necesario darle una mirada a los resultados de la propuesta por factores.

Se observan tres tipos de resultados. Uno de ellos tiene un porcentaje bastante bajo (24\%) que está expresado en el factor 4 denominado uso de la información debido a que el estándar 9 de este factor genera y analiza información, alcanza un porcentaje de $17 \%$ el cual fue evaluado solo por el grupo de los académicos quienes realizaron los siguientes comentarios: "El factor no lleva el nombre adecuado; el nombre no guarda relación con el contenido, debería llamarse evaluación y monitoreo del proceso; debe ser una referencia del factor, ser más explicativo"; "habria que desagregarlo, es muy denso, abarca muchos temas en uno solo".

Por otro lado, se tiene dos factores cercanos al nivel aceptable, 1 y 3 . En el caso del factor 1 dirección institucional, el estándar 2 proyecto curriculat y, el
3 liderazgo participativo alcanzan potcentajes de $51 \%$ y $65 \%$ respectivamente. Los expertos realizan los siguientes comentarios acerca de la propuesta de IPEBA en este factor: "Incorpora un elemento que tiene que ver con la formación integral, la inclusión"; "Los mecanismos pueden ser evaluados". "Se puede constatar que brinda orientaciones para el desarrollo de estrategias pedagógicas"; "Se puede medir la participación y procesos para medir mecanismos".

En cuanto al factor 3, Trabajo conjunto con las familias y comunidad, el estándar 8 (trabajo conjunto familia y comunidad en el diseño de estrategias), alcanza el porcentaje del $63 \%$. Al respecto, los expertos realizan los siguientes comentatios: "Se ve más coherencia, habla de la familia como de los actores de la comunidad".

Las opiniones, en los dos últimos factores presentados, son más o menos coincidentes pues ven la realidad educativa desde diferentes perspectivas.

Un tercer grupo de factores ( 2 y 5 ) son los que sobrepasaron el nivel de concordancia. En el factor 2 , denominado soporte al desempeño docente, es el estándar 5, implementación, el que alcanza un porcentaje de $96 \%$. Este valor es muy significativo parael resultado obtenido en este factor. Las opiniones de los expertos son las siguientes: "El indicador es coherente con el estándar. Mediante la utilización de los mapas de progreso, se asegura el proceso"; "La tmatriz se basa en la medición de estándares"; "Existe una coherencia vertical y horizontal porque se contará con programaciones progresivas"; "Se puede evaluar con una ficha de análisis de documento y ficha comparativa"." "Existe coherencia interna con el desempeño de los estudiantes en relación a las competencias de los estudiantes en las áreas curriculares"; "La evaluación del indicador se realiza de manera cualitativa".

El factor 5, llamado infraestructura y recursos para el aprendizaje, presenta el estándar 12 que gestiona a los recursos que dan soporte a la mejora del proceso de enseñanza aprendizaje con un porcentaje de $90 \%$ el mismo que eleva el resultado del factor. Las opiniones de los expertos son las siguientes: " Tiene criterios específicos como la gestión de 
Alétheia 2015,3 (1) 77

recursos técnicos, financieros, de infraestructura, de equipamiento y de material pedagógico, relacionados con la implementación del plan de mejora, lo que permite su evaluación".

"Es medible, tiene criterios especificos para evaluar el estándar". Estos factores por ser observables y de total competencia de los directores sobrepasan el nivel de concordancia aceptable $(60 \%)$ establecido como criterio.

\section{Conclusiones}

Las principales conclusiones de la investigación son las siguientes:

1. En términos generales, la propuesta de IPEBA no alcanzo el nivel de concordancia establecido como aceptable (60\%); sin embargo, se encuentra muy cerca $(57.05 \%)$, es decir, a $2.95 \%$ del criterio.

2. El factor 4, llamado uso de información, se encuentra muy por debajo con el $24.44 \%$.

3. Dosfactoresmuycercanosalniveldeconcordancia son el factor 1 (dirección institucional) con $53.80 \%$ y el factor 3 (Trabajo conjunto con las familias y comunidad) con $56.66 \%$.

4. Dos factores obturieron resultados muy por encima del nivel aceptable. Estos son el factor 2 (Soporte al desempeño docente) con $75.23 \%$ y el factor 5 (infraestructura y recursos para el aprendizaje) con $75.16 \%$.

5. El factor 4 (uso de información) merece sex revisado dado el resultado obtenido.

\section{Referencias}

Álvatez, I. (2006). Introducción a La calidad. Aproximación a los Sistemas de gestion y herramientas de calidad. Madrid, España: Ideas propias S. L. Recuperado de books.googlecom.pe/ books?isbn $=8498393604$

García de la Torre, M y otros. (2001). Modelo europeo de excelencia. Adaptación a los centros educativos del modelo de Fundación para la gestión de catidad. Madrid, España. Recuperado de http:// books.google.es/books?id=DENAGziysD
$\mathrm{gC} \& \mathrm{pg}=\mathrm{PA} 11 \& \mathrm{dq}=$ modelos + para $+\mathrm{la}+\mathrm{g}$ esti $\%$ C $3 \% \mathrm{~B} 3 \mathrm{n}+$ de + la + calidad +educativa $\&$ hl $=$ es\&sa $=$ X\&ei $=z 8 b E U c-h G d W t 4 A O$ gnoHYDQ\&ved=0CEEQ6AEwAQ\#v= onepage \&q $=$ modelos $\% 20$ para $\% 201 \mathrm{la} \% 20$ gesti $\% \mathrm{C} 3 \% \mathrm{~B} 3 \mathrm{n} \% 20 \mathrm{de} \% 20 \mathrm{la} \% 20$ calidad $\% 20$ educativa\& $\mathrm{f}=$ false

Heisenberg, E. (2008). Conceptos de educación. Recuperado de http://es.scribd.com/ doc/51750709/CONCEPTOS-DEEDUCACION

Morgan, P. (2011). IPEBA expone propuesta para acreditación de CETPRO, ante grupo de empresarios de CAPLAB. Recupetado de http://www. ipeba.gob.pe/index.php?option=com_con tent $\&$ view $=$ article\&id $=293$ :ipeba-exponepropuesta-para-acreditacion-de-cetproante-grupo-de-empresarios-decaplab\&catid $=368$ \& temid $=59$

Morgan, P. (2011). Matriz de evaluación para la arreditación de la calidad de la gestion educativa de instituciones de educación básica regutlar. Lima. Perú: Instituto Peruano de Evaluación, Acreditación y Certificación de la Calidad de la Educación Básica (IPEBA)

Ishikawa, K. (1986). ¿Qué es el control total de calidad? La modalidad japonesa. Bogotá, Colombia: Norma.

Bermeo, C. R (2012) Metaevaluación de la calidad de los estándares de acreditación de las carretas profesionales de educación según el modelo peruano (Tesis de doctorado).

Cano, E (1998). Evaluación de la calidad educativa. Madrid, España: Muralla S.A. Recuperado de http://books.google.es/books?id=abRZcOA 2ycMC\&printsec $=$ frontcover $\& \mathrm{dq}=\mathrm{calidad}+\mathrm{e}$ ducativa\&hl=es\&sa $=$ X\&ei=peafUZrEN4uu $8 \mathrm{AS} 7 \mathrm{qIBQ} \& v e d=0 \mathrm{CDQQ} 6 \mathrm{AEwAA \#} \mathrm{v}=$ on epage\&q $=$ calidad $\% 20$ educativa $\& \mathrm{f}=$ false 